\title{
A nail gun injury
}

\author{
Alisha Perkins • David A. Peak
}

Received: 10 December 2008 / Accepted: 12 May 2009/Published online: 4 August 2009

(C) Springer-Verlag London Ltd 2009

A 36-year-old right-handed male construction worker presented 2 days after feeling a sudden pain in his right maxillary area while using a nail gun. At that time, he thought that the nail gun flew up and hit him in the face without discharging a nail. He noted only mild pain and a small abrasion on his right cheek. Over the subsequent days he developed progressively worse pain and swelling of his right maxilla and eye as well as a right visual field cut. He was seen by an ophthalmologist who referred the patient to our ED.

On exam the patient had significant chemosis and rightsided palpebral edema with moderate ptosis. Further examination demonstrated decreased range of motion of the right eye in all directions and a right nasal hemianopsia. A CT scan of the head revealed a nail penetrating through the maxillary sinus and orbit missing the optic nerve and ophthalmic arteries and extending into the right frontal lobe (Figs. 1, 2, 3 and 4). He was taken to the operating room and had the nail removed by neurosurgery and plastic surgery. Follow-up ophthalmology examination on postoperative day 2 demonstrated temporal macular choroidal rupture responsible for the nasal hemianopsia with pre-retinal fibrosis and hemorrhage causing macular traction and retinal folds with vision 20/160 (compared to $20 / 25$ of the unaffected eye) and a complaint of a mild headache.

A. Perkins $(\bowtie) \cdot$ D. A. Peak

Department of Emergency Medicine,

Massachusetts General Hospital,

55 Fruit Street,

Boston, MA, USA

e-mail: aperkins1@partners.org

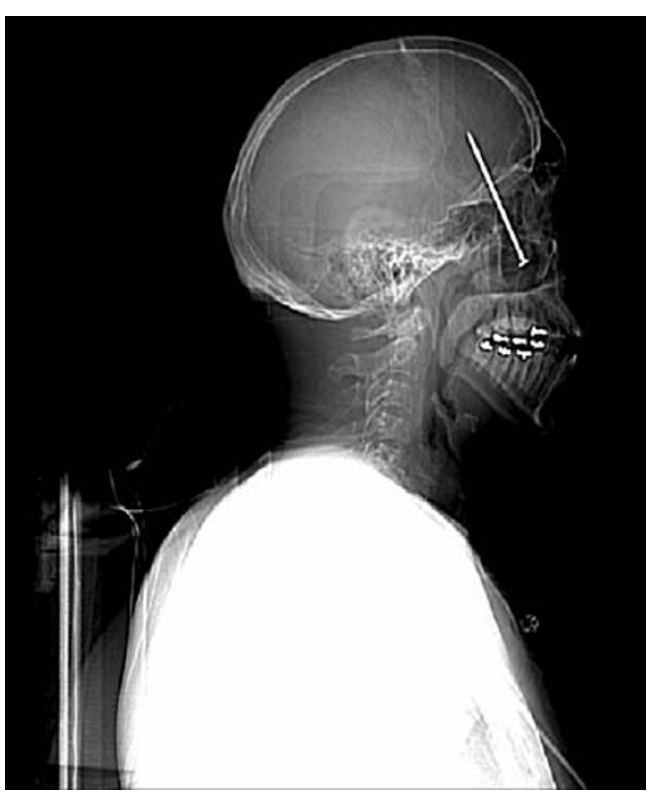

Fig. 1 Lateral skull $\mathrm{X}$-ray showing a nail traversing through the maxillary sinus to the skull base

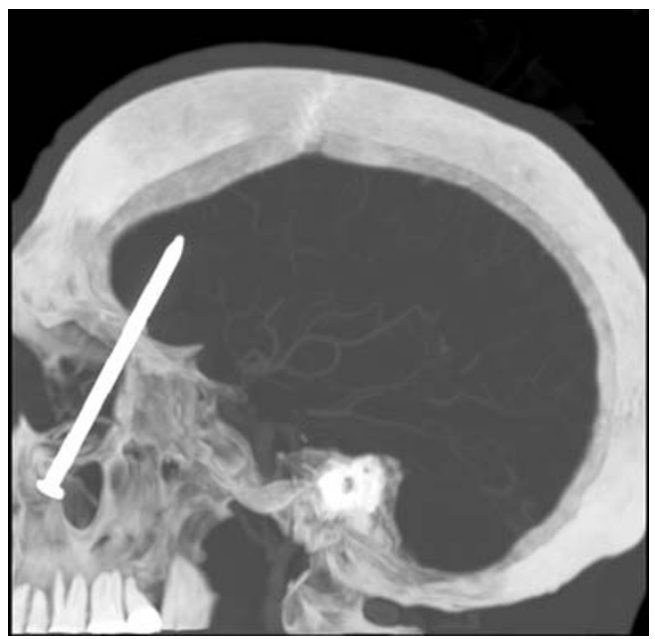

Fig. 2 Sagittal head CT with the bone window showing the implanted nail 


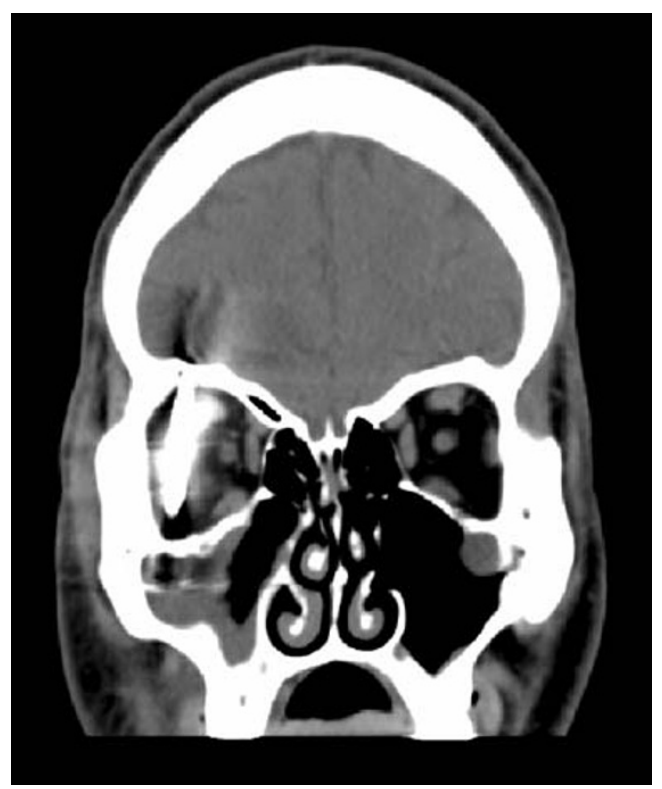

Fig. 3 Coronal head $\mathrm{CT}$ with the brain window showing the implanted nail

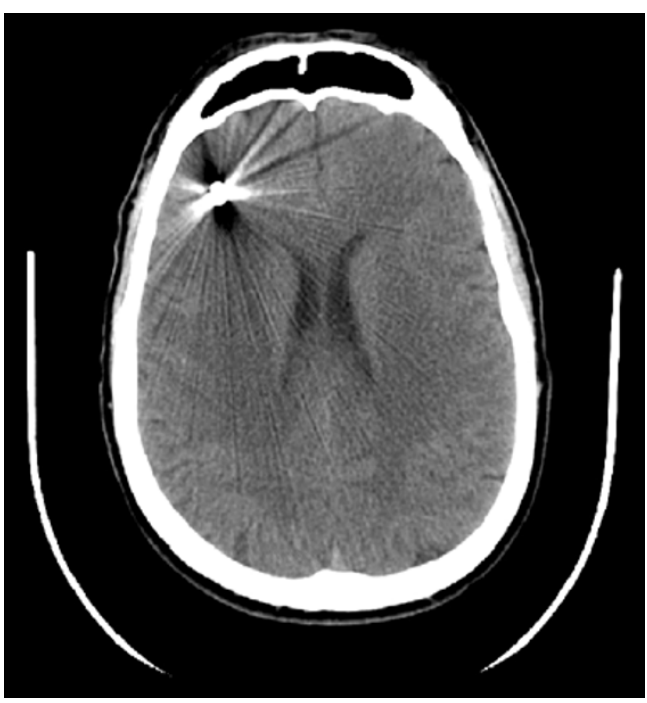

Fig. 4 Axial head CT with the brain window showing the nail in the right frontal lobe 